\title{
Verringerung der Emission von Thermoholz durch nachträgliche Behandlung mit Ammoniak
}

\author{
E. Roffael • R. Kraft • P. Niemz
}

Eingegangen: 7. Januar 2011 / Online publiziert: 21. Dezember 2011

(C) The Author(s) 2011. Dieser Artikel ist auf Springerlink.com mit Open Access verfügbar

Zusammenfassung Thermisch modifiziertes Holz (TMT) gibt hohe Mengen an Aldehyden (Furfural und Formaldehyd) sowie an flüchtigen organischen Säuren (Ameisen- und Essigsäure) ab. Die Abgabe von Aldehyden und Säuren lässt sich mit Hilfe der Flaschenmethode (EN 717-3) bestimmen. Die Ergebnisse zeigen, dass die Emission an flüchtigen Säuren und Aldehyden durch nachträgliche Ammoniakbehandlung erheblich reduziert wird. Der pH-Wert der Absorptionsflüssigkeit im Flaschenverfahren erhöht sich durch die Ammoniakbehandlung. Dies ist ein Hinweis auf die Abpufferung der Säuren im Holz.

\section{Reduction of emissions from thermally modified timber (TMT) through treatment with ammonia}

\begin{abstract}
Thermally modified timber (TMT) emits a high amount of aldehydes (furfural, formaldehyde) and volatile organic acids (formic and acetic acids). The release of aldehydes and acids, as assessed by the flask-method (EN 7173 ), can be reduced significantly by ammonia fumigation. The emission of formaldehyde is attenuated by more than $90 \%$. Emission of furfuraldehyde decreased in most cases
\end{abstract}

\footnotetext{
E. Roffael ( $\varangle)$

Georg-August-Universität Göttingen, Büsgenweg 1,

Göttingen 37077, Deutschland

e-mail: eroffae1@gwdg.de

R. Kraft

Abteilung Molekulare Holzbiotechnologie und Technische

Mykologie, Büsgen-Institut der Universität Göttingen, Göttingen,

Deutschland

e-mail: rkraft2@gwdg.de

P. Niemz

ETH Zürich, Schafmattstr. 6, 8093 Zürich, Switzerland

e-mail:niemzp@ethz.ch
}

by more than $70 \%$. The $\mathrm{pH}$-value of TMT increased due to ammonia fumigation above the neutral point indicating buffering of the volatile acids.

\section{Einleitung}

Die thermische Behandlung des Holzes hat als Methode der Holzvergütung in den letzten 10 Jahren industriell an Bedeutung gewonnen (Ewert und Scheiding 2005; Hill 2006). Durch die thermische Behandlung wird die Sorptionsfeuchte des Holzes herabgesetzt, die Dimensionsstabilität erhöht und die biologische Resistenz verbessert. Für die thermische Behandlung sind inzwischen mehrere Verfahren entwickelt worden, die sich unterschiedlicher Prinzipien bedienen.

Während der thermischen Behandlung bei Temperaturen von $160^{\circ} \mathrm{C}-220^{\circ} \mathrm{C}$ erfährt das Holz tiefgreifende Veränderungen in seinen chemischen Eigenschaften (Sandermann und Augustin 1964; Kollmann und Fengel 1965; Fengel 1966a, 1966b; Windeisen et al. 2009). Zu den chemischen Veränderungen gehört die Bildung von Zersetzungsprodukten aus den Neben- und Hauptkomponenten des Holzes, die zu Emission von flüchtigen organischen Verbindungen aus dem Holz führt (Roffael et al. 2007, 2008). So wiesen Schäfer und Roffael (2000) nach, dass die Extraktstoffe in Kiefern- und Fichtenholz die Abgabe von Formaldehyd bei der thermischen Behandlung des Holzes unter thermohydrolytischen Bedingungen fördern. Ferner stellten Roffael et al. (2007) fest, dass die thermische Behandlung von monomeren Zuckern, die bei der thermohydrolytischen Behandlung des Holzes entstehen (Sohn und Lenel 1949) zur Bildung von Formaldehyd und Furfural führt; die Mengen an Furfural und Formaldehyd fielen je nach Art der monomeren Zucker unterschiedlich aus. Die Bildung von Formal- 
dehyd, Essig- und Ameisensäure wurde ebenfalls für industriell hergestelltes Thermoholz der Fichte, Buche und Esche von Roffael et al. (2008) nachgewiesen, die darüber hinaus feststellten, dass das Verfahren der Herstellung von Thermoholz für die Emission an Säuren eine besondere Rolle spielt und die Mengen an entstehenden Emissionen mit beeinflusst.

Thermohölzer werden in verschiedenen Anwendungen eingesetzt, die eine Verminderung der Emission erfordern, hierzu gehören der Fensterbau und der Fußbodendielenbereich. Parkett und Fußböden aus thermisch modifiziertem Holz benötigen ab dem 01.01.2011 eine allgemeine bauaufsichtliche Zulassung in Form einer Emissionsprüfung nach dem AgBB-Schema (Scheiding 2011). Es ist deshalb von Interesse, die Abgabe von flüchtigen Verbindungen aus Thermoholz zu verringern. Dies trifft vor allem für die Abgabe an Furfural, Formaldehyd, insbesondere aber von Ameisenund Essigsäure zu, die in relativ großen Mengen entstehen. Graf et al. (2005) haben quantitative Messungen von Essigsäure, Furfural und Terpenen an Thermohölzern verschiedener Baumarten (Eiche, Esche, Lärche, Robinie und Tanne) ermittelt, wobei Essigsäure dominierte. Über Methoden zur Verringerung der Emission von Thermoholz liegen bislang, soweit bekannt, kaum Ergebnisse von systematischen Untersuchungen vor.

\section{Zielsetzung der Arbeit}

Diese Arbeit zielte darauf ab, durch nachträgliche Behandlung mit gasförmigem Ammoniak, das bei der Zersetzung von Ammoniumcarbonat entsteht, die Emission von flüchtigen Säuren (Ameisen- und Essigsäure) einzudämmen. Ferner sollte festgestellt werden, inwieweit auch durch die gleiche Behandlung die Abgabemengen an Formaldehyd und Furfural verringert werden können. Das Verfahren soll später im industriellen Maßstab erprobt werden.

Als Grundlage für die Vorgehensweise dient die Erfahrung der Holzwerkstoffindustrie. Die Ammoniakbehandlung von Holzspanplatten, die mit Aminoplastharzen als Bindemittel hergestellt sind, ist im Schrifttum (vgl. Roffael und Menzel 1980) und in der Patentliteratur beschrieben (DE-OS 2903254, DE-OS 2804514). Hierfür sind mehrere Verfahren vorgeschlagen worden, die sich zwar nicht im chemischen Wirkungsprinzip unterscheiden, aber in der Vorgehensweise bei der Behandlung. Eine ausführliche Abhandlung über die nachträgliche Behandlung von Holzspanund Holzfaserplatten ist bereits veröffentlicht worden (vgl. Roffael 1993). Nach Roffael und Menzel (1980) beträgt die Abnahme der Formaldehydabgabe nach Ammoniakbegasung und Lagerung für drei Monate je nach verwendetem Bindemittel zwischen etwa $55 \%$ und $70 \%$.

Neben den Erfahrungen der Holzwerkstoffindustrie mit der Ammoniakbehandlung ist die Wirkung von Ammoni-

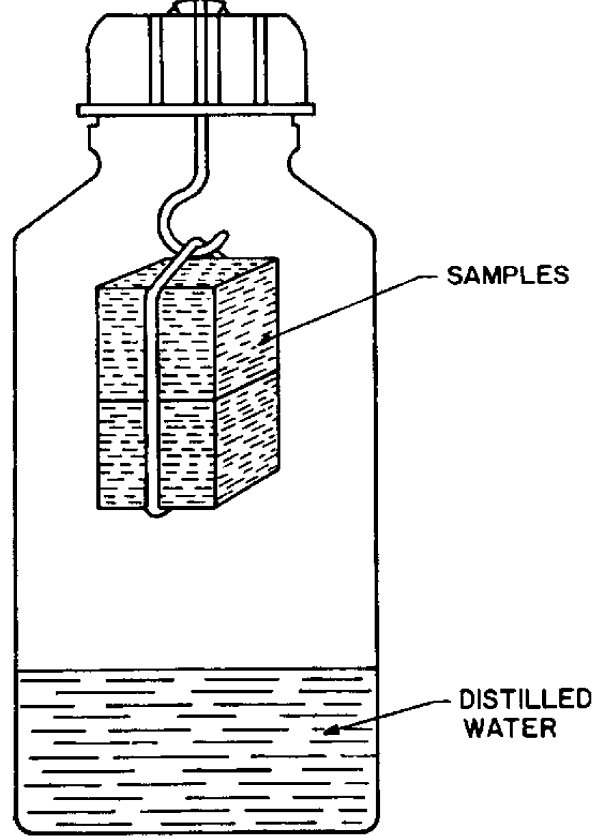

Abb. $1500 \mathrm{ml}$ PE-Weithalsflasche mit $50 \mathrm{ml}$ destilliertem Wasser und Probenmaterial (EN-717-3)

Fig. $1500 \mathrm{ml}$ PE-bottle with $50 \mathrm{ml}$ distilled water (bottom) and samples hanging above (EN 717-3)

ak auf das Holz selbst ebenfalls bekannt. Bereits vor etwa vier Jahrzehnten hat Bariska (1969) einen Übersichtsartikel geschrieben, in dem die bis dahin erschienene Literatur besprochen wurde. Neuere Arbeiten über die Wirkung der Ammoniakbegasung auf das Holz haben Besold und Fengel (1983a, 1983b, 1983c) durchgeführt. Ammoniakbehandlung von thermisch unbehandeltem Holz führt zur Bildung von Essigsäure, Acetamid und Ammoniak, wie kürzlich von Aehlig et al. (2011) festgestellt wurde. Demgegenüber liegen über die Sekundäremissionen beim Thermoholz nach einer Behandlung mit Ammoniak, soweit bekannt, keine publizierten Angaben vor.

\section{Versuchsdurchführung}

Untersucht wurden industriell nach dem Feuchte-WärmeDruck (FWD)-Verfahren hergestellte Thermohölzer der Holzarten Buche und Fichte. Für die Untersuchungen wurden aus dem Thermoholz Prüfkörper mit den Abmessungen von ca. $25 \mathrm{~mm} \times 25 \mathrm{~mm} \times 20 \mathrm{~mm}$ ausgeformt. Anschließend wurde ein Teil der Probekörper in einem geschlossenen Behälter für $72 \mathrm{~h}$ über $10 \mathrm{~g}$ Ammoniumcarbonat bei Raumtemperatur gelagert, so dass die Proben mit Ammoniak begast 
Abb. 2 Formaldehydabgabe, gemessen nach der

Flaschenmethode (24 h-Wert unbehandeltem und mit Ammoniak begastem

Thermoholz der Fichte und

Buche

Fig. 2 Formaldehyde release, 717-3), from untreated and ammonia treated thermally modified timber (TMT) after

Abb. 3 Furfuralabgabe, Flaschenmethode (24 h-Wert und $48 \mathrm{~h}$-Wert), von unbehandeltem und mit Ammoniak begastem Thermoholz der Fichte und Buche

Fig. 3 Furfural release, assessed by the flask method (EN 717-3), from untreated and ammonia treated thermally modified timber (TMT) after testing time of $24 \mathrm{~h}$ and $48 \mathrm{~h}$ und $48 \mathrm{~h}$-Wert), von assessed by flask method (EN testing time of $24 \mathrm{~h}$ and $48 \mathrm{~h}$ gemessen nach der
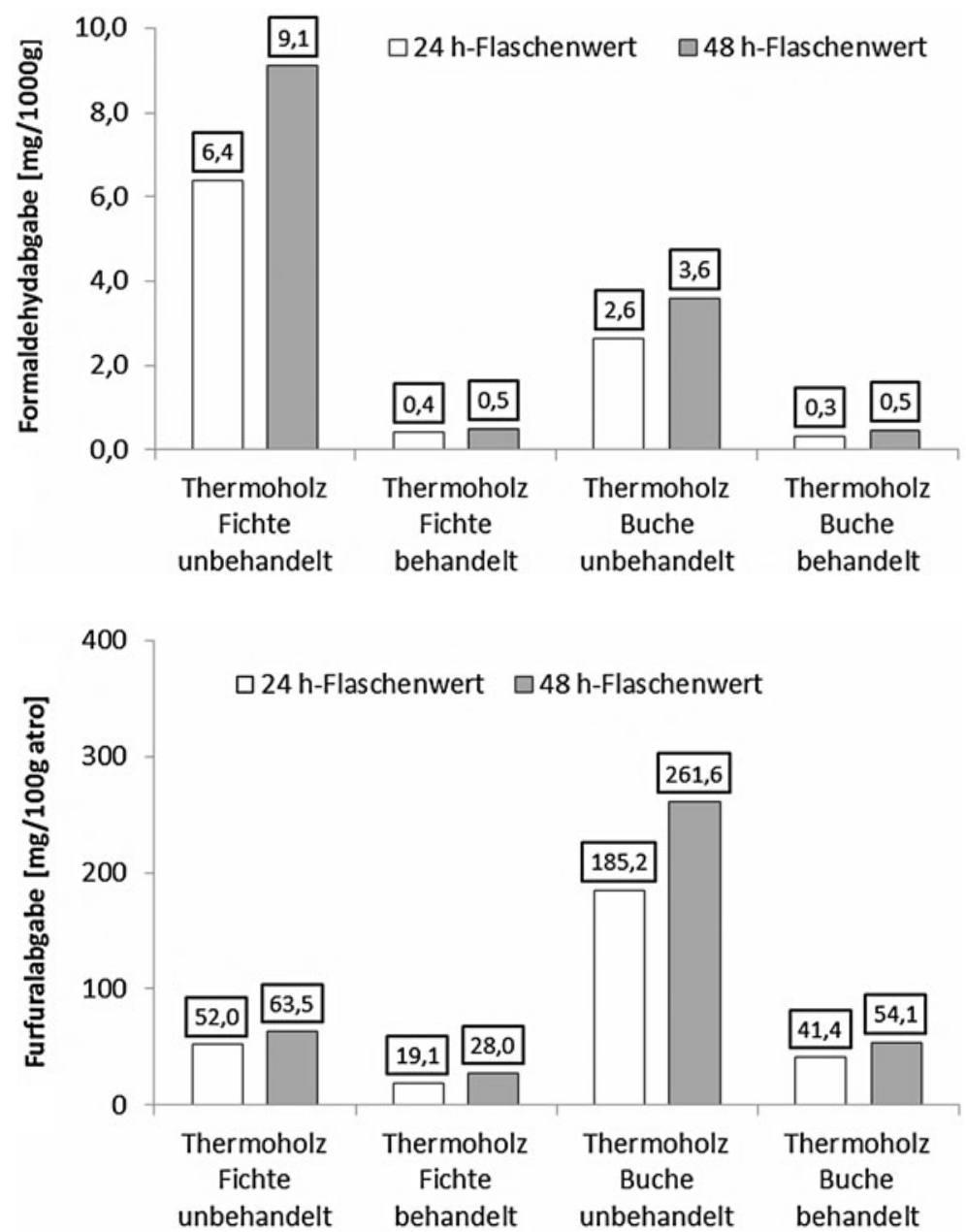

wurden. Nach dieser Behandlung wurden die Prüfkörper für ca. $48 \mathrm{~h}$ belüftet. Ein anderer Teil der Prüfkörper blieb unbehandelt.

Nach diesem Schritt wurden sowohl die mit Ammoniak behandelten, als auch die unbehandelten Thermoholzproben (jeweils 2 Klötzchen, ca. 17 g) auf ihre Emissionen untersucht. Hierzu wurde das Flaschenverfahren in Anlehnung an EN 717-3 (Abb. 1) verwendet, wobei das Verfahren dahingehend „modifiziert“ wurde, dass die Flaschen für $24 \mathrm{~h}$ und $48 \mathrm{~h}$ bei $40^{\circ} \mathrm{C}$ im Trocknungsschrank gelagert wurden. Die abgegebenen Mengen an Formaldehyd, Furfural sowie Ameisen- und Essigsäure wurden durch Analyse der Absorptionsflüssigkeit bestimmt. Hierbei wurde der abgegebene Formaldehyd nach der Acetylacetonmethode (EN 7173) erfasst. Die Furfuralkonzentration wurde in der Absorptionsflüssigkeit photometrisch bei einer Wellenlänge von $278 \mathrm{~nm}$ ermittelt. Mit ähnlichen Ergebnissen ist mit Hilfe der Stenhouse-Reaktion zu rechnen (vgl. Saad 1965). Die Analyse der Säuren erfolgte ionenchromatographisch. Ferner wurde jeweils der pH-Wert der Absorptionsflüssigkeit mit einem laborüblichen $\mathrm{pH}-$ Meter bestimmt. Grundsätzlich wurden bei den Messungen von jeder Holzprobe Doppelbestimmungen durchgeführt.

\section{Ergebnisse und Diskussion}

Die Ergebnisse der Untersuchungen sind in Abb. 2 (Formaldehydabgabe), Abb. 3 (Furfuralabgabe), Abb. 4 (pH-Wert), Abb. 5 (Abgabe an Ameisensäure) und Abb. 6 (Abgabe an Essigsäure) zusammengestellt. Die Ergebnisse zeigen, dass die Begasung des Thermoholzes mit Ammoniak über Ammoniumcarbonat die Formaldehydabgabe aus den hergestellten Thermohölzern erheblich reduziert. Die Abnahme der Formaldehydabgabe durch die Ammoniakbegasung war im Falle von Fichtenholz besonders ausgeprägt. Hierfür dürften zwei Faktoren verantwortlich sein; zum einen kann Formaldehyd mit Ammoniak unter Bildung von Hexamethylentetramin (Hexa) reagieren, das nicht wasserdampfflüchtig ist. Zum anderen wird durch die Ammoniakbehandlung der $\mathrm{pH}$-Wert des Holzes erheblich verschoben und zwar über den neutralen Punkt $(\mathrm{pH}=7)$ hinweg in den schwach alkalischen $\mathrm{pH}-\mathrm{Bereich}$ hinein. Im alkalischen $\mathrm{pH}$-Bereich ist Formaldehyd kaum wasserdampfflüchtig und auch Hexamethylentetramin, wenn überhaupt, unter den herrschenden Randbedingungen kaum zersetzbar. Die Verringerung der Formaldehydabgabe von Holzwerkstoffen durch nachträgliche Ammoniakbegasung ist bekannt (DE-OS 2903254). In- 
Abb. 4 pH-Wert in der wässrigen

Absorptionsflüssigkeit der Flaschenmethode nach einer Reaktionsdauer von $24 \mathrm{~h}$ und $48 \mathrm{~h}$, von unbehandeltem und mit Ammoniak begastem

Thermoholz der Fichte und

Buche

Fig. $4 \mathrm{pH}$-value in the aqueous absorption solution of untreated and ammonia treated thermally modified timber (TMT) after a reaction period of $24 \mathrm{~h}$ and $48 \mathrm{~h}$ in the flask method (EN 717-3)
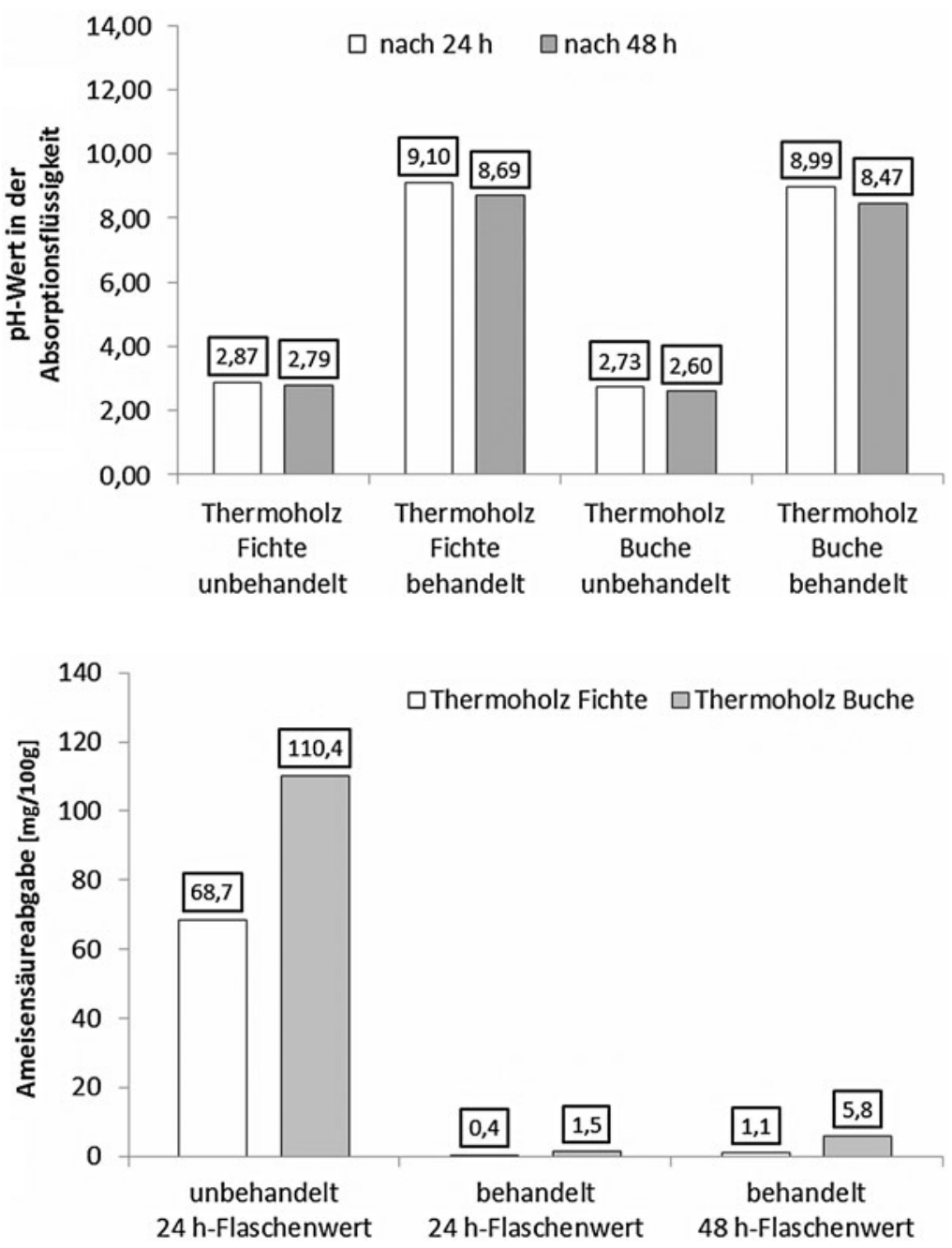

Abb. 5 Ameisensäureabgabe in der Absorptionsflüssigkeit von unbehandeltem Buchen- und Fichtenholz (nach einer Prüfdauer von $24 \mathrm{~h}$ im Flaschenverfahren) sowie von mit Ammoniak begastem Thermoholz (nach einer Behandlungsdauer von $24 \mathrm{~h}$ und $48 \mathrm{~h}$ ) der Buche und Fichte

Fig. 5 Release of formic acid from untreated (after a reaction period of $24 \mathrm{~h}$ ) and ammonia treated thermally modified timber (TMT) of spruce and beech (after a reaction period of $24 \mathrm{~h}$ and $48 \mathrm{~h}$ )
$24 \mathrm{~h}$-Flaschenwert

als Neutralisationsmittel bei der thermohydrolytischen Behandlung von Kiefernholz untersuchten und dabei feststellten, dass die Ammoniakzugabe zu dem Faserstoff, wie zu erwarten, die entstehenden flüchtigen Ameisen- und Essigsäure stark abpuffert. Es ist ferner nicht völlig auszuschließen, dass das Ammoniak im Holz auch mit dem Lignin reagiert. Flaig et al. (1959) stellten einen durchschnittlichen Stickstoffgehalt von etwa $20 \%$ nach Behandlung von Sulfitablaugen mit Ammoniak bei $130^{\circ} \mathrm{C}$ in wässriger Lösung fest. Außerdem ist davon auszugehen, dass Ammoniak im Holz zum Teil adsorbiert vorliegt.

In Abb. 5 und Abb. 6 sind die Ergebnisse der Abgabe an Ameisen- und Essigsäure aus dem Thermoholz vor und nach einer Behandlung mit Ammoniakgas zusammengestellt. Daraus wird deutlich, dass durch die Ammoniakbehandlung die Abgabe an Ameisensäure weitaus stärker verringert wird als die der Essigsäure. Die Ursache dafür liegt womöglich darin, dass Ameisensäure eine stärkere Säure $(\mathrm{pKa}=3,7)$ als Essigsäure $(\mathrm{pKa}=4,8)$ ist. Durch Erhöhung des $\mathrm{pH}$-Wertes reagiert die Ameisensäure weitaus stärker mit dem Ammoniak als die Essigsäure. Durch die Re- 
Abb. 6 Essigsäureabgabe in der Absorptionsflüssigkeit, von unbehandeltem Buchen- und Fichtenholz (nach einer Prüfdauer von $24 \mathrm{~h}$ im

Flaschenverfahren) und von mit Ammoniak begastem

Thermoholz (nach einer Reaktionsdauer von $24 \mathrm{~h}$ und 48 h) der Buche und Fichte

Fig. 6 Release of acetic acid from untreated (after a reaction period of $24 \mathrm{~h}$ ) and ammonia treated thermally modified timber (TMT) of spruce and beech (after a reaction period of $24 \mathrm{~h}$ and $48 \mathrm{~h}$ )

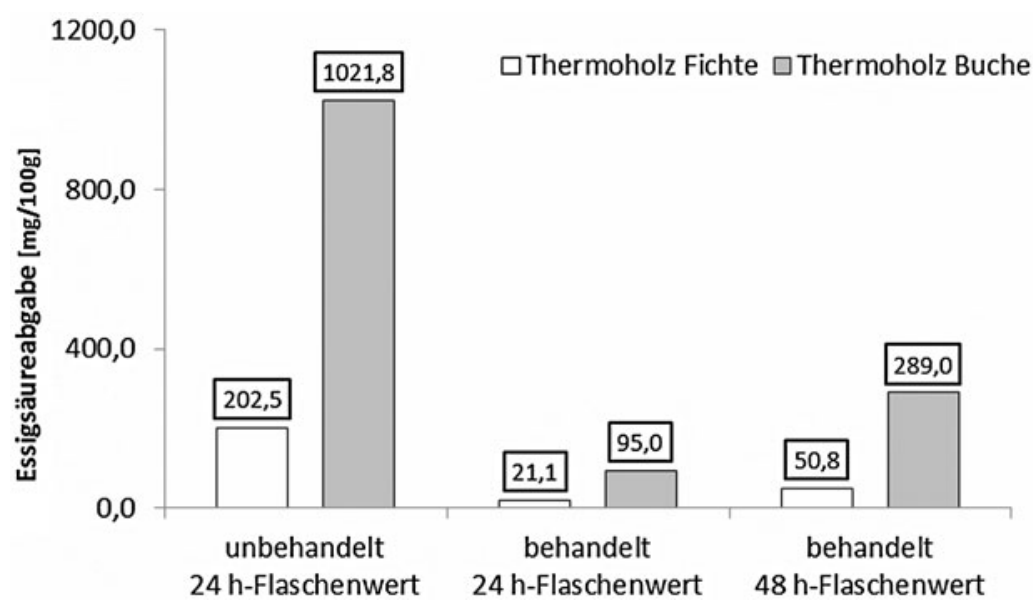

Fengel D (1966b) Über die Veränderung des Holzes und seiner Komponenten im Temperaturbereich bis $200^{\circ} \mathrm{C}$. Teil 2 - Die Hemicellulosen in unbehandeltem und thermisch behandeltem Fichtenholz. Holz Roh- Werkst 24:98-109

Flaig W, Hingst KP, Wesselhoeft P (1959) Verfahren zur Herstellung von stickstoffreichen Ligninprodukten. Deutsches Patent Nr. DE-AS 1745632

Graf N, Wagner S, Begander U, Trinkaus P, Boechzelt H (2005) Gaseous emissions from thermally wood modification as a source for fine chemicals recovery. Joanneum Research $\mathrm{GmbH}$, Graz

Hill CAS (2006) Wood modification, chemical, thermal and other processes. Wiley, Chichester. ISBN-13: 978-0-470-02172-9

Kollmann F, Fengel D (1965) Änderung der chemischen Zusammensetzung von Holz durch thermische Behandlung. Holz RohWerkst 23:461-468

Nicewicz D, Onisko W (1987) Ammoniak als Neutralisationsmittel im Prozess der thermo-mechanischen Zerfaserung von Holz. Holzforsch Holzverwert 39(4):89-94

Roffael E, Menzel (1980) Holzspanplatten - Aminoplastharze Ammoniakbehandlung - Formaldehydabgabe, WKI-Kurzbericht $22 / 80$

Roffael E (1993) Formaldehyde release from particleboards and other wood based panels. Malaysian forest records Nr. 37

Roffael E, Hameed M, Kraft R (2007) Bildung von Formaldehyd, Furfural und Ameisensäure bei der thermohydrolytischen Behandlung von einigen monomeren Zuckern (Xylose, Arabinose und Galactose). Holztechnologie 48(3):15-18

Roffael E (2008) On the release of volatile acids from wood-based panels-chemical aspects. Holz Roh- Werkst 66:373-378

Roffael E, Kraft R, Niemz P (2008) Formaldehyd und Säureabgabe aus Thermoholz. Holz-Zentbl 134(50):1418

Saad M (1965) Beiträge zur titrimetrischen und spectrophotometrischen Pentosanbestimmung. Dipl.-Arbeit an der Fakultät für Chemie, Biologie, Geologie und Mineralogie der Technischen Hochschule Darmstadt

Sandermann W, Augustin H (1964) Chemische Untersuchungen über die thermische Zersetzung von Holz. 3. Mitteilung. Holz RohWerkst 22:373-386

Schäfer M, Roffael E (2000) On the formaldehyde release of wood. Holz Roh- Werkst 58(4):259-264

Scheiding W (2011) Mündliche Mitteilung an E Roffael am 06.01.2011

Sohn AW, Lenel PO (1949) Über die Reaktionsprodukte einer schonenden partiellen Hydrolyse. Papier 3:109-118

Windeisen E, Bächle H, Zimmer B, Wegener G (2009) Relation between chemical changes during the production of thermo-treated beech wood. Holzforschung 63:773-778 